\title{
TEM CHARACTERIZATION OF SINGLE CRYSTAL SILICON FILM ON GLASS SUBSTRATE
}

\author{
S. C. Cheng and K. P. Gadkaree
}

Science and Technology, Corning Incorporated, Corning, NY 14831

Recently a new process was developed to obtain single crystal silicon film on glass substrate (SOG) at Corning Inc. [1]. The process not only allows the formation of a thin single crystal silicon film on the glass substrate, but also creates a silica interface layer and a barrier layer in the glass, which is permanently free of any mobile ions.

At various stages of the above project, cross-sectional TEM samples were prepared to characterize the chemistry and the crystallinity properties of the transferred thin films, and the interface between the film and the glass substrate.

Figure 1 is a bright field image, taken from the sample prepared by implant $\mathrm{H}^{+}$. The image clearly shows two regions in the Si film. On the top region of the film, the crystal structure is significantly damaged. The exfoliated Si layer containing the damaged layer has bonded well to the glass substrate. Figure 2 is the enlarged image of the damaged area. Many microcracks are observed from the image. It is believed that these cracks were developed during the annealing process from the $\mathrm{H}^{+}$related crystal defects, such as $\mathrm{Si}-\mathrm{H}$ defect in the system or high pressurized $\mathrm{H}_{2}$ gas bubbles. The $\mathrm{Si}-\mathrm{H}$ defects were observed in silicon wafer before the bonding procedures. Figure 3 is a bright field image of these defects on Si wafer. The orientation of the specimen was tilted away from (011) to increase the contrast of the defects in the image. The thickness fringes, shown in the image, cross the whole areas of specimen except the damaged layer. No microcracks similar to that, shown in Fig. 2 were observed in Fig. 3. The microcracks in the Si wafer after annealing, shown in Fig. 2, play an important role in separating the film from the Si mother wafer. Figure 4 is a bright field image, which reveals that there are two layers in the interface region between the silicon film and the glass substrate. EDS and EELS techniques were used to analyze the chemical distribution and stoichiometry of the interfacial layers. The first interfacial layer is a silica layer, and the second is a barrier layer, in which mobile ions are not present. Details of the results will be presented at a separated paper [1].

In order to reveal possible roughness at atomic scale and possible lattice defects at the interface, a high magnification HRTEM image of the interface was taken and shown in Fig. 5. The Si crystal was at $<011>$ orientation. The image shows that the (200) planes are parallel to the interface and the (111) planes form a $55^{\circ}$ angle with the interface. In Fig. 5, the roughness of the interface is estimated to be about $0.5 \mathrm{~nm}$. Also grain boundaries are absent in the HRTEM image and it is an evidence that the Si film on the substrate is a single crystal.

Acknowledgements

The authors gratefully acknowledge John Grazul, Matthew Weyland and David Muller for using the facilities at CCMR of Cornell University. 


\section{Reference}

1. Kishor P. Gadkaree and et al. to be published.
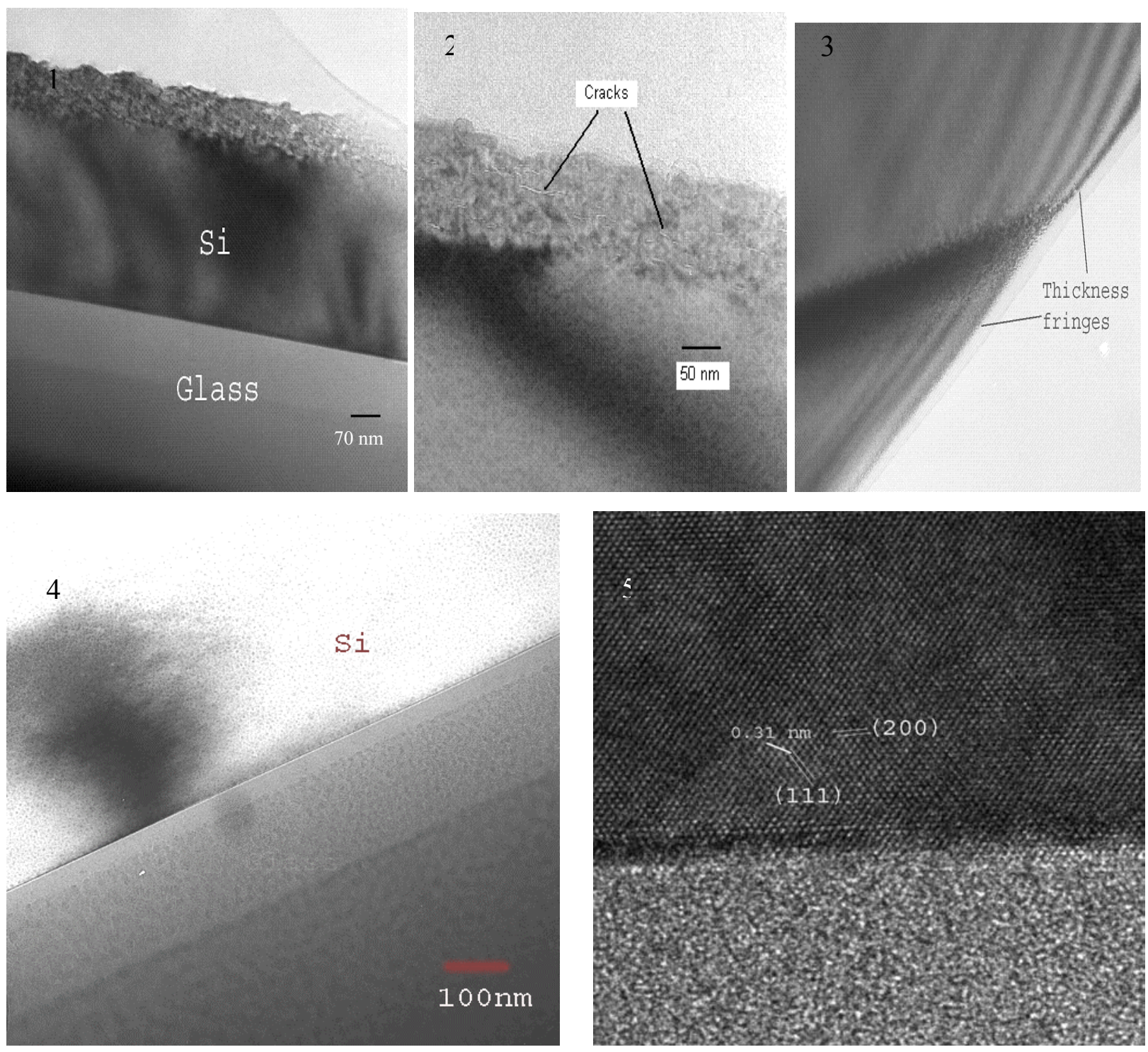

Fig. 1. Bright field image of sample prepared by implant $\mathrm{H}^{+}$at $100 \mathrm{keV}$. The image clearly shows two regions in the Si film.

Fig. 2. Enlarged image of Fig. 1 on the damaged area to show the microcracks.

Fig. 3. Bright field image of the defects in Si wafer before bounded to glass substrate.

Fig. 4. Bright field image of interfacial region for EDS and EELS study.

Fig. 5. HRTEM image of the single crystal Si film and the interface between the film and the glass substrate. 